\title{
La neuroeducación en el aprendizaje de los estudiantes de la I.E.S.P.P. Didascalio «Jesús Maestro» de Santiago, Cusco 2015
}

\author{
Yanina Rojas Anaya \\ Universidad Andina del Cusco \\ yrojas@uandina.edu.pe
}

\section{Resumen}

Una de las características más resaltantes que encontramos en la deficiencia educacional, es el desconocimiento por parte de los docentes de los nuevos aportes de la neuroeducación y cómo este podría mejorar el aprendizaje de los estudiantes.

Objetivo: Diseñar un taller en neuroeducación para ser aplicado en el aprendizaje de los estudiantes de la I.E.S.P.P. Didascalio «Jesús Maestro» 2015.

Tipo y diseño de investigación: descriptivo no experimental o ex post facto.

Conclusiones: Es necesario conocer el funcionamiento del cerebro y aplicarlo en el aprendizaje, ya que el estudio del cerebro es fundamental en la educación y los docentes deben ser conscientes de su uso, por lo cual, deben aplicarlo en sus sesiones de clase.

Palabras clave: Neuroeducación, aprendizaje, estilos de aprendizaje, inteligencias múltiples, inteligencia emocional, PNL, pensamiento crítico.

\footnotetext{
Abstract

The neuroeducation in the learning of students of I.E.S.P.P. Didascalio "Jesus Maestro" of Santiago, Cusco 2015

One of the most striking features found in educational deficiency is the lack of knowledge about teachers the new contributions of neuroeducation, and how this could improve student learning. Objective: To propose a workshop on neuroeducation to be applied in the learning of students of the I.E.S.P.P. Didascalio "Jesus Maestro" 2015.

Type and research design: descriptive non-experimental or ex post facto.

Conclusions: It is necessary to know the functioning of the brain and to apply it in the learning, since the study of the brain is fundamental in the education and the teachers must be aware of its use, therefore, they must apply it in their class sessions.
} 
Keywords: Neuroeducation, learning, learning styles; multiple intelligences, emotional intelligence, NLP. critical thinking.

\section{Introducción}

La educación es parte fundamental en el desarrollo de un país, por tal razón, se hace necesario que todo docente — de cualquier nivel - maneje las estrategias necesarias para lograr que los estudiantes logren los objetivos propuestos en su formación y, más allá, sean conscientes del desarrollo integral de la persona y, por ende, del futuro profesional como formador. Ya que no basta con brindar y exigir que se tengan los conocimientos necesarios ni desarrollar las competencias seleccionadas, sino que es necesario desarrollar potencialidades (inteligencia emocional, PNL, estilos de aprendizaje, pensamiento crítico), que solo se pueden fortalecer con la aplicación de la neuroeducación, debido a que esta permitirá que la persone logre un aprendizaje permanente, el mismo que vaya de la mano con la investigación y la creatividad, además de buscar el razonamiento y la autonomía con criterio propio.

\section{MATERIALES Y MÉTODOS}

\subsection{Tipo y diseño de estudio}

Se realizó un estudio descriptivo de diseño no experimental durante el 2015. El estudio fue revisado y aprobado por la Unidad de Segunda Especialidad de la Universidad Nacional de San Agustín de Arequipa, sede Cusco.

\subsection{Población y muestra}

La población estuvo constituida por 50 estudiantes de la I.E.S.P.P. Didascalio «Jesús Maestro» del Distrito de Santiago, durante el 2015 


\subsection{Operacionalización de las variables}

\begin{tabular}{|c|c|c|c|}
\hline VARIABLES & ÍNDICE & DEFINICIÓN & INDICADOR \\
\hline \multirow{5}{*}{ Neuroeducación } & $\begin{array}{l}\text { Inteligencia } \\
\text { emocional }\end{array}$ & $\begin{array}{l}\text { Habilidad para atender y } \\
\text { percibir sentimientos de forma } \\
\text { apropiada y precisa, regulando } \\
\text { o modificando nuestro estado } \\
\text { de ánimo y el de los demás. }\end{array}$ & $\begin{array}{c}\text { Autoconocimiento } \\
\text { Comprensión de emociones } \\
\text { Estilos de pensamiento }\end{array}$ \\
\hline & $\begin{array}{l}\text { Inteligencias } \\
\text { múltiples }\end{array}$ & $\begin{array}{l}\text { Capacidad de todos los seres } \\
\text { humanos para conocer el } \\
\text { mundo de modos diferentes. }\end{array}$ & $\begin{array}{l}\text { Lingüística, musical, kinésica } \\
\text { natural, espacial, interpersonal } \\
\text { lógico matemática }\end{array}$ \\
\hline & $\begin{array}{c}\text { Programación } \\
\text { neurolingüística }\end{array}$ & $\begin{array}{l}\text { Clave para modificar nuestros } \\
\text { valores, emociones y formas de } \\
\text { expresarnos para así poder } \\
\text { cambiar la percepción de la } \\
\text { realidad y la autoimagen de las } \\
\text { personas. }\end{array}$ & $\begin{array}{c}\text { Escucha activa } \\
\text { Asertividad } \\
\text { Rapport } \\
\text { Claridad en la comunicación }\end{array}$ \\
\hline & $\begin{array}{c}\text { Estilo de } \\
\text { aprendizaje }\end{array}$ & $\begin{array}{l}\text { Forma de cómo cada persona } \\
\text { utiliza su propio método o } \\
\text { estrategia para aprender }\end{array}$ & $\begin{array}{l}\text { Reflexivo } \\
\text { Pragmático }\end{array}$ \\
\hline & $\begin{array}{l}\text { Pensamiento } \\
\text { crítico }\end{array}$ & $\begin{array}{l}\text { Es la habilidad para analizar y } \\
\text { evaluar la consistencia de los } \\
\text { razonamientos. }\end{array}$ & $\begin{array}{ll}\text { Interpretación } & \text { Análisis } \\
\text { Evaluación } & \text { Inferencia } \\
\text { Explicación } & \\
\text { Autorregulación } & \end{array}$ \\
\hline $\begin{array}{c}\text { V2 } \\
\text { Aprendizaje }\end{array}$ & $\begin{array}{c}\text { Conceptual } \\
\text { Procedimental } \\
\text { Actitudinal }\end{array}$ & $\begin{array}{l}\text { Conjunto saberes para } \\
\text { determinar el grado de } \\
\text { conocimiento, el saber hacer, y } \\
\text { el grado de valores. }\end{array}$ & $\begin{array}{l}\text { Registro de notas } \\
\text { Lista de cotejo } \\
\text { Escala de estimación }\end{array}$ \\
\hline
\end{tabular}




\subsection{Técnicas e instrumentos}

Para recolectar datos se procedió de la siguiente manera:

a) Análisis de documentos: se analizaron diversos documentos (tesis de investigación, textos en físico y virtuales) para desarrollar y proponer talleres basados en neuroeducación y aplicarlo en el aprendizaje de los estudiantes.

b) Entrevista: Se realizó una encuesta a cada miembro de la muestra (estudiantes). La encuesta estuvo validada por dos docentes de la Universidad San Agustín de Arequipa.

\subsection{Resultados}

Los resultados a los que se llegó, se determinaron sobre la base del conocimiento que tienen los estudiantes (futuros docentes, tanto del nivel inicial como primaria) del I.E.S.P.P. Didascalio «Jesús Maestro» sobre neuroeducación. A continuación se muestra la encuesta aplicada:

Las respuestas que se dieron en la encuesta indican que, pese a que muchos escucharon hablar de neuroeducación, estilos de aprendizaje, PNL, etc.; casi siempre lo emplean en clase, lo que equivale a un $37,50 \%$; mientras que, el $62,50 \%$ no aplica ningún tipo de conocimiento en neuroeducación. Esto se puede sintetizar en el siguiente cuadro: 


\begin{tabular}{|c|c|c|c|}
\hline \multicolumn{4}{|l|}{ Encuesta } \\
\hline & \multicolumn{2}{|l|}{ Sí } & \multirow[t]{2}{*}{ No } \\
\hline 1. ¿Conoce los planteamientos de la neuroeducación? & & & \\
\hline \multicolumn{4}{|l|}{ SI RESPONDIÓ “SÍ”, CONTINÚE CON LAS DEMÁS PREGUNTAS: } \\
\hline & Nunca & $\begin{array}{l}\text { Algunas } \\
\text { veces }\end{array}$ & siempre \\
\hline \multicolumn{4}{|l|}{ 2. ¿Hace uso de los planteamientos de la neuroeducación? } \\
\hline \multicolumn{4}{|l|}{$\begin{array}{l}\text { 3. ¿Desarrolla el autoconocimiento en el proceso de aprendizaje de } \\
\text { sus estudiantes? }\end{array}$} \\
\hline \multicolumn{4}{|l|}{$\begin{array}{l}\text { 4. ¿Desarrolla la comprensión de emociones en sus estudiantes } \\
\text { durante el proceso de aprendizaje? }\end{array}$} \\
\hline \multicolumn{4}{|l|}{ 5. ¿Aplica la escucha activa durante el proceso de aprendizaje? } \\
\hline \multicolumn{4}{|l|}{ 6. ¿Desarrolla la asertividad en sus estudiantes? } \\
\hline \multicolumn{4}{|l|}{ 7. ¿Emplea el rapport en el desarrollo de sus sesiones de clase? } \\
\hline \multicolumn{4}{|l|}{$\begin{array}{l}\text { 8. ¿Promueve la claridad en la comunicación durante sus actividades } \\
\text { de enseñanza- aprendizaje? }\end{array}$} \\
\hline \multicolumn{4}{|l|}{ 9. ¿Identifica los estilos de aprendizaje de sus estudiantes? } \\
\hline $\begin{array}{l}\text { 10. ¿Emplea los estilos de aprendizaje durante el proceso de enseñanza } \\
\text {-aprendizaje de sus estudiantes? }\end{array}$ & & & \\
\hline
\end{tabular}

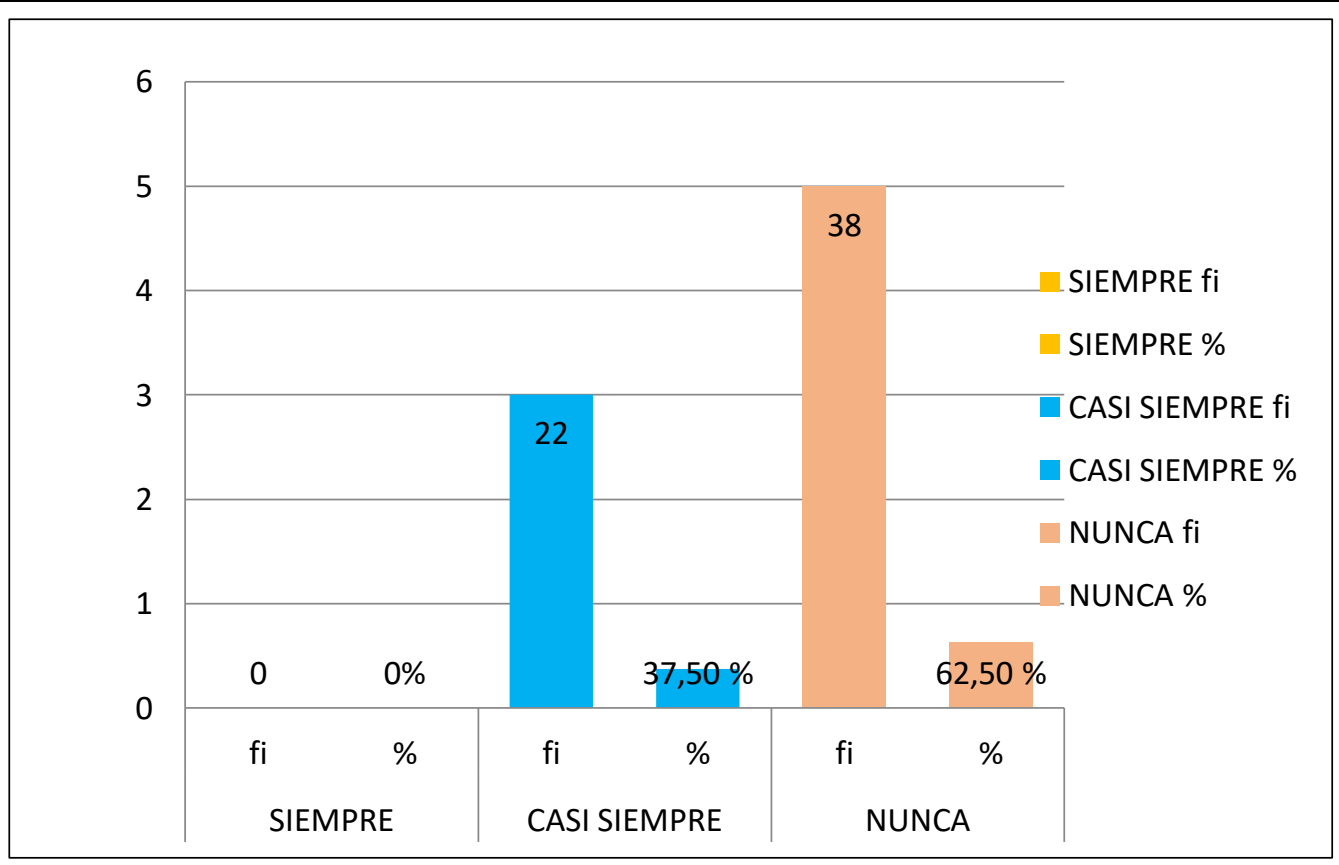




\subsection{Discusión}

La presente investigación tuvo como objetivo general, diseñar un taller en neuroeducación para ser aplicado en el aprendizaje de los estudiantes de la I.E.S.P.P. Didascalio «Jesús Maestro» 2015. Se aprecia que la mayoría de estudiantes (futuros docentes), sí conoce lo que es la 'neuroeducación', pero un gran porcentaje de estudiantes $(62,50 \%)$ no lo aplica, esto quiere decir, que más de la mitad de la población se caracteriza por desconocer la parte práctica de este tema.

Los resultados hallados por Izaguirre en su estudio sobre la aplicación de conocimientos neurocientíficos en el aula y la mejora de la calidad percibida del servicio educativo en la Escuela Profesional de Turismo y Hotelería de la USMP, 2010 indican la mejora significativa en un $57 \%$, lo cual se pudo verificar en las sesiones aplicadas de los docentes, demostrándose que sí es importante, no solo conocer, sino también aplicar la neuroeducación en las sesiones de clase para mejorar el aprendizaje (Izaguirre Sotomayor, 2011).

Asimismo, debemos indicar que es necesario considerar los aportes de la neurociencia a la educación, porque lo estudiantes de esta generación ya no son iguales al de otras generaciones, así en la investigación presentada por Marcos, se determinó que la forma de enseñanza es aún errónea por lo que, el aprendizaje presenta un nivel bajo; todavía los profesores enseñan de manera tradicional, no aplican las nuevas propuestas de la neuroeducación, como son estilos de aprendizaje y de pensamiento, inteligencia emocional, PNL, etc. (Marcos Sánchez, 2012). Lo ideal sería que en cada escuela, existiera un núcleo de profesores de ciencias naturales, de humanidades, de artes, etc., que trabajaran en conjunto para conocer más y profundizar más en la teoría del aprendizaje compatible con el cerebro.

Estos trabajos corroboran la importancia de la neuroeducación en la formación de futuros docentes, razón por la cual se hace imprescindible que, tanto docentes como estudiantes, profundicen los aportes de esta nueva disciplina para aplicarlo en la enseñanza y aprendizaje. 


\subsection{Conclusiones}

1. La neuroeducación busca conocer el funcionamiento del cerebro y aplicarlo en el aprendizaje y enseñanza. Todas las acciones que realizamos son producto de una actividad cerebral, de ahí la importancia de conocer el cerebro.

2. El estudio del cerebro es fundamental en el proceso de enseñanzaaprendizaje, ya que el ser humano se desenvuelve como producto de su sistema nervioso. Si los neurotransmisores funcionan bien, esto repercutirá en el comportamiento y, por ende, en el aprendizaje. Asimismo, los estilos de aprendizaje y de pensamiento: la PNL y la inteligencia emocional se determinan en función al estudio cerebral.

3. Los docentes deben ser conscientes de que la generación de estos últimos años es diferente y tienen otras habilidades; razón por la cual, es deber del docente conocer a profundidad esos cambios para llegar a los estudiantes y ayudar a ser más competentes.

4. Es necesario que los docentes conozcan en qué consiste la neuroeducación (inteligencias múltiples, inteligencia emocional, PNL, estilos de aprendizaje y pensamiento crítico) y aplicarlo en sus sesiones de clase, de esta manera, el aprendizaje mejorará.

5. La mayor parte de docentes — pese a indicar que conocen aspectos de la neuroeducación - solo lo identifican de manera teórica, mas no lo llevan a la práctica como por ejemplo, los estilos de aprendizaje; pero la aplicación de este y otros temas no lo saben desarrollar en clase de modo pleno. 


\subsection{Recomendaciones}

Una vez que se ha determinado en qué consiste la neuroeducación, nos permitimos realizar las siguientes sugerencias que deben ser tomadas en cuenta:

1. Desarrollar talleres y cursos sobre neuroeducación para mejorar el aprendizaje de los estudiantes.

2. Los docentes de todas las áreas deben emplear los conocimientos de la neuroeducación, como, por ejemplo: identificar los estilos de aprendizaje y enseñar en base a lo identificado, desarrollar la inteligencia emocional, la asertividad, etc., durante el proceso de enseñanza.

\subsection{Diseño de un taller para conocer la neuroeducación y aplicarlo en las sesiones de clase}

\section{A. Fundamento}

Como es necesario que toda investigación deba tener carácter científico, se necesita vincular la teoría con la práctica. Si bien muchos conocen el tema de neuroeducación, no en su totalidad identifican todos los temas que esta encierra, así como tampoco lo utilizan para desarrollar sus sesiones de clase. Por tal razón, se propone la implementación de talleres para fomentar la neuroeducación en la enseñanza.

Es necesario que todos tomemos conciencia de que la generación de ahora se diferencia en muchos aspectos de generaciones anteriores, por eso, no solo basta con enseñar sino también mirar más allá del educando, tomar en cuenta sus estilos de aprendizaje, estilos de pensamiento, su tipo de inteligencia.

\section{B. Objetivos}

\section{Objetivo general}

Diseñar un taller en neuroeducación para ser aplicado en el aprendizaje de los estudiantes de la I.E.S.P.P. Didascalio «Jesús Maestro» 2015. 


\section{Objetivos específicos:}

- Reconocer la importancia de la neuroeducación y su aplicación en la enseñanza y aprendizaje en el I.E.S.P.P. Didascalio «Jesús Maestro» 2015.

- Identificar los estilos de aprendizaje de los alumnos del I.E.S.P.P. Didascalio «Jesús Maestro» 2015.

- Identificar los estilos de pensamiento de los estudiantes del I.E.S.P.P. Didascalio «Jesús Maestro» 2015.

- Determinar la importancia de la PNL del I.E.S.P.P. Didascalio «Jesús Maestro» 2015.

- Proporcionar a los estudiantes del I.E.S.P.P. Didascalio «Jesús Maestro» 2015 una guía pedagógica sobre neuroeducación.

\section{Recursos}

$\rightarrow$ Recursos humanos: estudiantes del I.E.S.P.P. Didascalio «Jesús Maestro.»

$\rightarrow$ Recursos materiales: textos de investigación, pizarra, plumones.

\section{Plan de lección}




\section{Talleres de capacitación}

\begin{tabular}{|c|c|c|c|c|c|}
\hline $\begin{array}{l}\text { SESI } \\
\text { ÓN }\end{array}$ & TEMA & OBJETIVO & ACTIVIDADES & $\begin{array}{l}\text { TIEM } \\
\text { PO }\end{array}$ & RECURSOS \\
\hline 1 & $\begin{array}{c}\text { Bases } \\
\text { teóricas de la } \\
\text { neuro } \\
\text { educación }\end{array}$ & $\begin{array}{l}\text { Analizar la } \\
\text { neuroeducación } \\
\text { como base de la } \\
\text { enseñanza y } \\
\text { aprendizaje. }\end{array}$ & $\begin{array}{l}\text { Presentación de expositores y de } \\
\text { los temas a desarrollar durante el } \\
\text { taller. } \\
\text { Esclarecer el concepto y las } \\
\text { bases teóricas de la } \\
\text { neuroeducación y su incidencia } \\
\text { en la enseñanza y aprendizaje. }\end{array}$ & $4 \mathrm{hrs}$ & \multirow{9}{*}{$\begin{array}{c}\text { Retroproyector } \\
\text { Cañón } \\
\text { multimedia }\end{array}$} \\
\hline 2 & $\begin{array}{l}\text { Los neuro } \\
\text { trasmisores }\end{array}$ & $\begin{array}{l}\text { Identificar los } \\
\text { neurotransmisores y } \\
\text { su influencia en el } \\
\text { aprendizaje }\end{array}$ & $\begin{array}{l}\text { Clasificación de los } \\
\text { neurotransmisores y } \\
\text { reconocimiento de su influencia } \\
\text { en el aprendizaje. }\end{array}$ & & \\
\hline 3 & PNL & $\begin{array}{l}\text { Analizar y } \\
\text { reflexionar sobre el } \\
\text { uso de la PNL }\end{array}$ & $\begin{array}{l}\text { Analizar las técnicas de la PNL } \\
\text { en el proceso de aprendizaje. }\end{array}$ & $4 \mathrm{hrs}$ & \\
\hline 4 & $\begin{array}{c}\text { Comunica } \\
\text { ción Asertiva }\end{array}$ & $\begin{array}{l}\text { Determinar la } \\
\text { importancia de la } \\
\text { comunicación } \\
\text { asertiva durante las } \\
\text { sesiones de clase. }\end{array}$ & $\begin{array}{l}\text { Analizar la importancia de la } \\
\text { asertividad en la comunicación. }\end{array}$ & $4 \mathrm{hrs}$ & \\
\hline 5 & $\begin{array}{l}\text { Estilos de } \\
\text { aprendizaje }\end{array}$ & $\begin{array}{l}\text { Identificar los estilos } \\
\text { de aprendizaje. }\end{array}$ & $\begin{array}{l}\text { Aprender a identificar los estilos } \\
\text { de aprendizaje. } \\
\text { Aplicar estrategias de enseñanza } \\
\text { según el estilo de aprendizaje. }\end{array}$ & $4 \mathrm{hrs}$ & \\
\hline 6 & $\begin{array}{c}\text { Estilos de } \\
\text { pensamiento }\end{array}$ & $\begin{array}{l}\text { Identificar los estilos } \\
\text { de pensamiento }\end{array}$ & $\begin{array}{l}\text { Identificar y aplicar estrategias } \\
\text { según el estilo de pensamiento. }\end{array}$ & $4 \mathrm{hrs}$ & \\
\hline 7 & $\begin{array}{l}\text { Inteligencias } \\
\text { múltiples }\end{array}$ & $\begin{array}{l}\text { Analizar las } \\
\text { inteligencias } \\
\text { múltiples en la } \\
\text { enseñanza - } \\
\text { aprendizaje. }\end{array}$ & $\begin{array}{l}\text { Identificar y aplicar estrategias } \\
\text { de acuerdo a las inteligencias } \\
\text { múltiples. }\end{array}$ & $4 \mathrm{hrs}$ & \\
\hline 8 & $\begin{array}{l}\text { Inteligencia } \\
\text { emocional }\end{array}$ & $\begin{array}{l}\text { Reflexionar acerca de } \\
\text { la importancia de la } \\
\text { inteligencia } \\
\text { emocional. }\end{array}$ & $\begin{array}{l}\text { Analizar la importancia de la } \\
\text { inteligencia emocional en el } \\
\text { proceso de aprendizaje. }\end{array}$ & $4 \mathrm{hrs}$ & \\
\hline 9 & $\begin{array}{l}\text { Evaluación } \\
\text { del taller }\end{array}$ & Cierre del taller & $\begin{array}{l}\text { Se realizará una lluvia de ideas } \\
\text { acerca de las dudas que se } \\
\text { tengan con respecto al taller. } \\
\text { Se les pedirá que respondan un } \\
\text { cuestionario para la evaluación } \\
\text { de los conocimientos. }\end{array}$ & $4 \mathrm{hrs}$ & \\
\hline
\end{tabular}




\section{BIBLIOGRAFÍA}

Izaguirre Sotomayor, M. H. (2011). La aplicación de conocimientos neurocientíficos en el aula y la mejora de la calidad percibida del servicio educativo. Lima: USMP. Obtenido de https://es.scribd.com/document/287963024/Aplicacion-de-Conocimientos-en-El-Aula-25-10

Marcos Sánchez, V. (2012). Salvando la brecha digital: La plasticidad del cerebro joven frente a los circuitos neuronales ya consolidados de un cerebro adulto. Salamanca: Fondo Editorial de la Universidad de Salamanca. Recuperado el 2 de agosto de 2017, de http://www.gabinetecomunicacionyeducacion.com/sites/default/files/field/adjuntos/salvando_la_ brecha_digital_la_plasticidad_del_cerebro_joven_frente_a_los_circuitos_neuronales_ya_consolida dos_de_un_cerebro_adulto.pdf 